\title{
Correction to: Self assembly and controlled drug release of a nano- laminated graphite carbon nitride/methotrexate complex
}

\author{
Shanshan Liu ${ }^{1}$ Zhaoliang Guo ${ }^{1}$ Xiongfeng Zeng ${ }^{1} \cdot$ Xianguang Meng $^{1} \cdot$ Haina Sun ${ }^{1} \cdot$ Yizao Wan² $\cdot$ Guifu Zuo ${ }^{1}$
}

Published online: 4 December 2018

(c) Springer Science+Business Media, LLC, part of Springer Nature 2018

\section{Correction to: Journal of Materials Science: Materials in Medicine (2018) 29: 116}

https://doi.org/10.1007/s10856-018-6128-3

The original version of this article unfortunately contained a mistake. In the Abstract section the sentence "Furthermore, the drug release mechanism was investigated by kinetic models and a first-order relationship was concluded, which indicated that the drug release is a simple diffusion process cohydroxyapatite/methotrexate complexntrolled by gradient drug concentration." was wrong. It should read as "Furthermore, the drug release mechanism was investigated by kinetic models and a first-order relationship was concluded, which indicated that the drug release is a simple diffusion process controlled by gradient drug concentration."
The original article can be found online at https://doi.org/10.1007/ s10856-018-6128-3.

Guifu Zuo

zuoguifu@163.com

1 Hebei Provincial Key Laboratory of Inorganic Nonmetallic Materials, College of Materials Science and Engineering, North China University of Science and Technology, 063210 Tangshan, Hebei, People's Republic of China

2 School of Materials Science and Engineering, Tianjin University, 300072 Tianjin, People's Republic of China 